\title{
Magnetic fields around AGB stars and Planetary Nebulae
}

\author{
W. H. T. Vlemmings ${ }^{1}$ \\ ${ }^{1}$ Department of Earth and Space Sciences, Chalmers University of Technology, Onsala Space \\ Observatory, SE-439 92 Onsala, Sweden
}

\begin{abstract}
Stars with a mass up to a few solar masses are one of the main contributors to the enrichment of the interstellar medium in dust and heavy elements. However, while significant progress has been made, the process of the mass-loss responsible for this enrichment is still not exactly known and forces beyond radiation pressure might be required. Often, the mass lost in the last phases of the stars life will become a spectacular planetary nebula. The shaping process of often strongly a-spherical PNe is equally elusive. Both binaries and magnetic fields have been suggested to be possible agents although a combination of both might also be a natural explanation.

Here I review the current evidence for magnetic fields around AGB and post-AGB stars pre-Planetary Nebulae and PNe themselves. Magnetic fields appear to be ubiquitous in the envelopes of apparently single stars, challenging current ideas on its origin, although we have found that binary companions could easily be hidden from view. There are also strong indications of magnetically collimated outflows from post-AGB/pre-PNe objects supporting a significant role in shaping the circumstellar envelope.
\end{abstract}

Keywords. polarization, magnetic fields, polarization, stars-AGB and post-AGB, planetary nebulae

\section{Introduction}

After the AGB phase, the stellar envelopes undergo a major modification as they evolve to Planetary Nebulae (PNe). The standard assumption is that the initial slow AGB mass loss quickly changes into a fast superwind, generating shocks and accelerating the surrounding envelope (Kwok et al. 1978). It is during this phase that the typically spherical circumstellar envelope evolves into a Planetary Nebula. As the majority of $\mathrm{PNe}$ are aspherical, an additional mechanism is needed to explain the departure from sphericity. Specifically the discovery that the collimated outflows of the pre-PNe (P-PNe), where such outflows are common, have a momentum that exceeds that which can be supplied by radiation pressure alone (Bujarrabal et al. 2001), has led to a revision of the standard theory. The formation mechanism of in particular bipolar PNe is still a matter of fierce debate. Current theories to explain the PNe shapes include binaries, disks, magnetic fields or a combination of these. A promising mechanism could be a binary companion or massive planet that helps maintain a strong magnetic field capable of shaping the outflow (e.g. Nordhaus et al. 2007). However, the known fraction of binary systems cannot yet explain the large number of aspherical PNe. Since the shaping mechanism is likely related to the mass loss mechanism responsible for the enrichment of the interstellar medium, a better understanding of this mechanism is crucial.

Here I will review the observational evidence for strong magnetic fields in PNe as well as around their AGB and post-AGB progenitors. I will give an overview of the methods that can be used to study magnetic fields, especially in light of the plethora of new instruments that will be available shortly. Finally, I will discuss a number of questions 


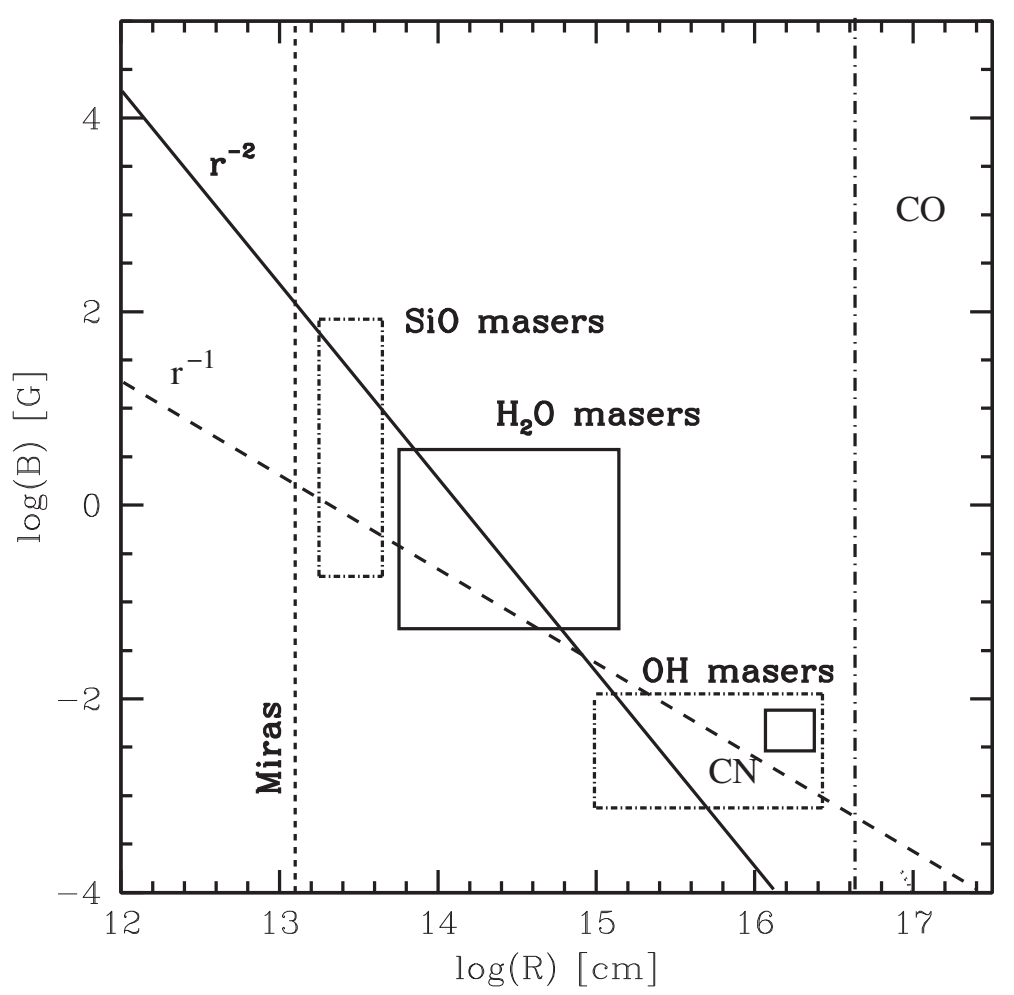

Figure 1. Magnetic field strength vs. radius relation as indicated by current maser polarization observation of a number of Mira stars. The boxes show the range of observed magnetic field strengths derived from the observations of $\mathrm{SiO}$ masers (Kemball et al. 2009, Herpin et al. 2006), $\mathrm{H}_{2} \mathrm{O}$ masers (Vlemmings et al. 2002, Vlemmings et al. 2005, Leal-Ferreira et al. 2013), $\mathrm{OH}$ masers (e.g. Rudnitski et al. 2010) and CN (Herpin et al. 2009). The thick solid and dashed lines indicate an $r^{-2}$ solar-type and $r^{-1}$ toroidal magnetic field configuration. The vertical dashed line indicates the stellar surface. ALMA CO polarization observations will uniquely probe the outer edge of the envelope (vertical dashed dotted line).

related this topic that we can expect to be answered with the new instruments in the next few years.

\section{Observational Techniques - Polarization}

With the exception of observations where the magnetic field strength is estimated assuming forms of energy equilibrium, such as synchrotron observations, the magnetic field strength and structure is typically determined from polarization observations.

\subsection{Circular Polarization}

Circular polarization, generated through Zeeman splitting, can be used to measure the magnetic field strength. It measures the total field strength when the splitting is large and the line-of-sight component of the field when the splitting is small. The predominant source of magnetic field strength information during the late stages of stellar evolution comes from maser circular polarization observations, and particularly the common SiO, $\mathrm{H}_{2} \mathrm{O}$ and $\mathrm{OH}$ masers. These can show circular polarization fractions ranging from $\sim 0.1 \%$ $\left(\mathrm{H}_{2} \mathrm{O}\right)$ up to $\sim 100 \%(\mathrm{OH})$ and are, because of their compactness and strength, excellent sources to be observed with high angular resolution. Unfortunately, the analysis of maser 

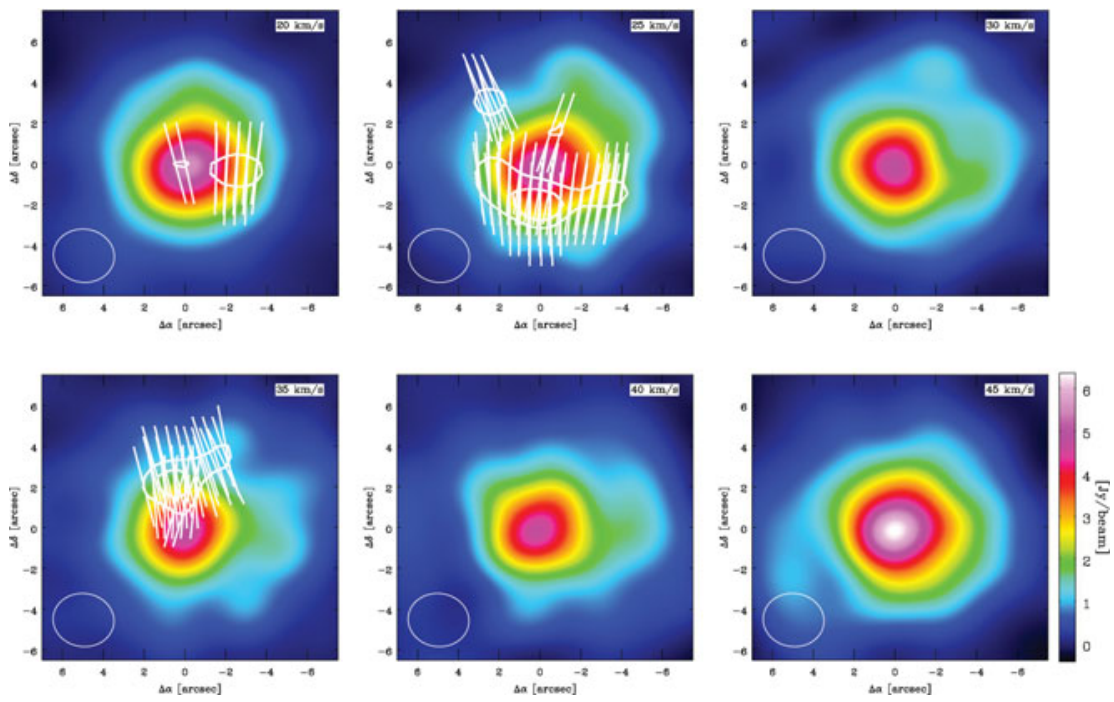

Figure 2. From Vlemmings et al. (2012), the polarization of the $\mathrm{CO}(2-1)$ line in the circumstellar envelope of IK Tau. The colors are the CO emission and the contours the linearly polarized intensity. The line segments indicate the electric vector polarization angle.

polarization is not straightforward (for a review, see Vlemmings (2012a)), and it has taken a long time before maser observations were acknowledged to provide accurate magnetic field measurements. More recently, the first attempts have been made to detect the Zeeman splitting of non-maser molecular lines, such as CN (Herpin et al. 2009). As many of these occur at shorter wavelength in the (sub-)mm regime, the advent of the Atacama Large (sub-)Millimeter Array will further enhance these types of studies.

\subsection{Linear Polarization}

Linear polarization, probing the structure of the plane-of-the-sky component of the magnetic field, can be observed both in the dust (through aligned grains) and molecular lines (through radiation anisotropy - the Goldreich-Kylafis effect). The Goldreich-Kylafis effect on CO has only recently been mapped for the first time in the envelope of evolved stars and will, with ALMA, allow for a systematic study of magnetic fields (Vlemmings et al. 2012b). Typical percentages of linear polarization range from up to a few percent (e.g. dust, $\mathrm{CO}, \mathrm{H}_{2} \mathrm{O}$ masers) to several tens of percent ( $\mathrm{OH}$ and $\mathrm{SiO}$ masers). Again the interpretation of maser polarization depends on a number of intrinsic maser properties, but in specific instances maser linear polarization can even be used to determine the full 3-dimensional field morphology. In addition to the geometry, the linear polarization of most notably dust, can also be used to obtain a value for the strength of the plane-ofthe-sky component of the magnetic field. This is done using the Chandrasekhar-Fermi method, which refers to the relation between the turbulence induced scatter of polarization vectors and the magnetic field strength.

\section{Current Status - Evolved Star Magnetic Fields}

\subsection{AGB Stars}

Most AGB magnetic field measurements come from maser polarization observations (SiO, $\mathrm{H}_{2} \mathrm{O}$ and $\mathrm{OH}$ ). These have revealed a strong magnetic field throughout the circumstellar envelope. In Figure 1, I have indicated the magnetic field strength in the regions of the 


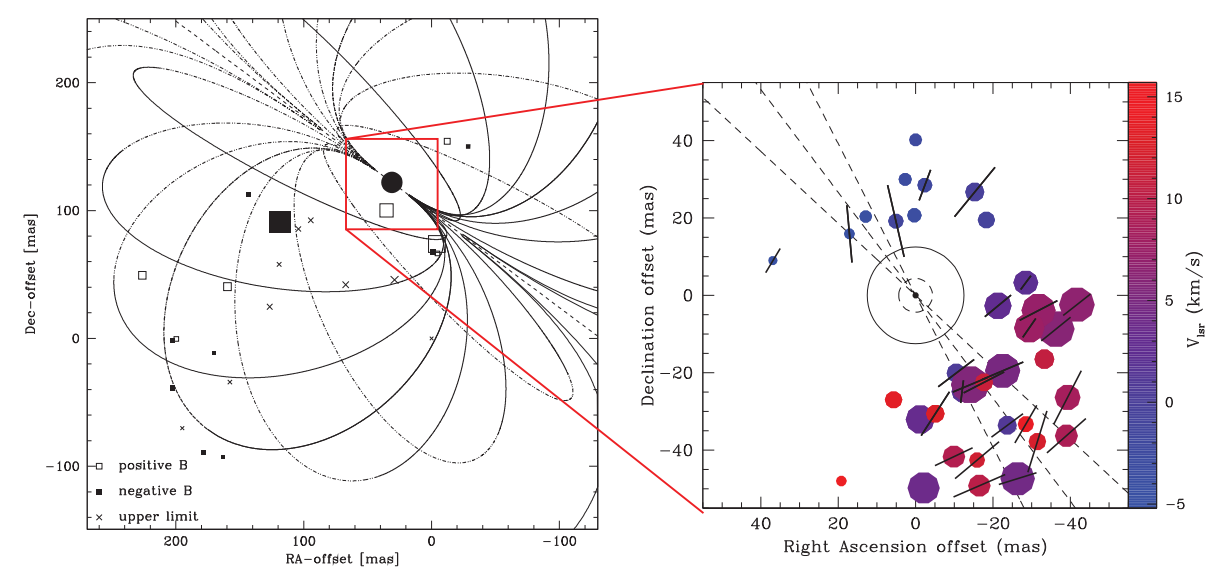

Figure 3. (left) The dipole magnetic field of the supergiant VX Sgr as determined from a fit to the $\mathrm{H}_{2} \mathrm{O}$ maser magnetic field observations (Vlemmings et al. 2005). (right) Positions and polarization of the VX Sgr $v=1, J=5-4{ }^{28} \mathrm{SiO}$ masers observed with the SMA (Vlemmings et al. 2011). The masers spots are plotted with respect to a weighted mean position of all maser spots. The black vectors are the observed polarization vectors scaled linearly according to polarization fraction. The long dashed inner circle indicates the star and the solid circle indicates the location of the $43 \mathrm{GHz} \mathrm{SiO}$ masers. The short dashed circle indicates the minimum radius of the ${ }^{28} \mathrm{SiO}$ masers. The dashed lines indicate the position angle and its uncertainty of the inferred orientation of the dipole magnetic field of VX Sgr observed using $\mathrm{H}_{2} \mathrm{O}$ and $\mathrm{OH}$ masers (Szymczak et al. 2001, Vlemmings et al. 2005).

envelope traced by the maser measurements throughout AGB envelopes. While a clear trend with increasing distance from the star is seen, the lack of accurate information on the location of the maser with respect to the central stars makes it difficult to constrain this relation beyond stating that it seems to vary between $B \propto R^{-2}$ (solar-type) and $B \propto R^{-1}$ (toroidal). Future observations of $\mathrm{CO}$ polarization might be able to provide further constraints.

As the masers used for these studies are mostly found in oxygen-rich AGB stars, it has to be considered that the sample is biased. However, recent CN Zeeman splitting observations (Herpin et al. 2009) seem to indicate that similar strength fields are found around carbon-rich stars.

Beyond determining the magnetic field strength, the large scale structure of the magnetic field is more difficult to determine, predominantly because the maser observations often probe only limited line-of-sights. Even though specifically $\mathrm{OH}$ observations seem to indicate a systematic field structure, it has often been suggested that there might not be a large scale component to the field that would be necessary to shape the outflow (Soker 2002). So far the only shape constraints throughout the envelope have been determined for the field around the supergiant star VX Sgr (Fig. 3), where maser observations spanning 3 orders of magnitude in distance are all consistent with a large scale, possibly dipole shaped, magnetic field.

However, the recent observations of the linear polarization of CO (and other molecular lines) caused by the Goldreich-Kylafis effect (Fig. 2, Vlemmings et al. 2012b) allows for a much more detailed study of the magnetic field morphology. In the case of IK Tau, the observations indicate a more or less uniform field from close to the star out to a few thousand AU. While the current sensitivity offered by the SMA is not sufficient for a more detailed magnetic field mapping, ALMA will revolutionize this field. 


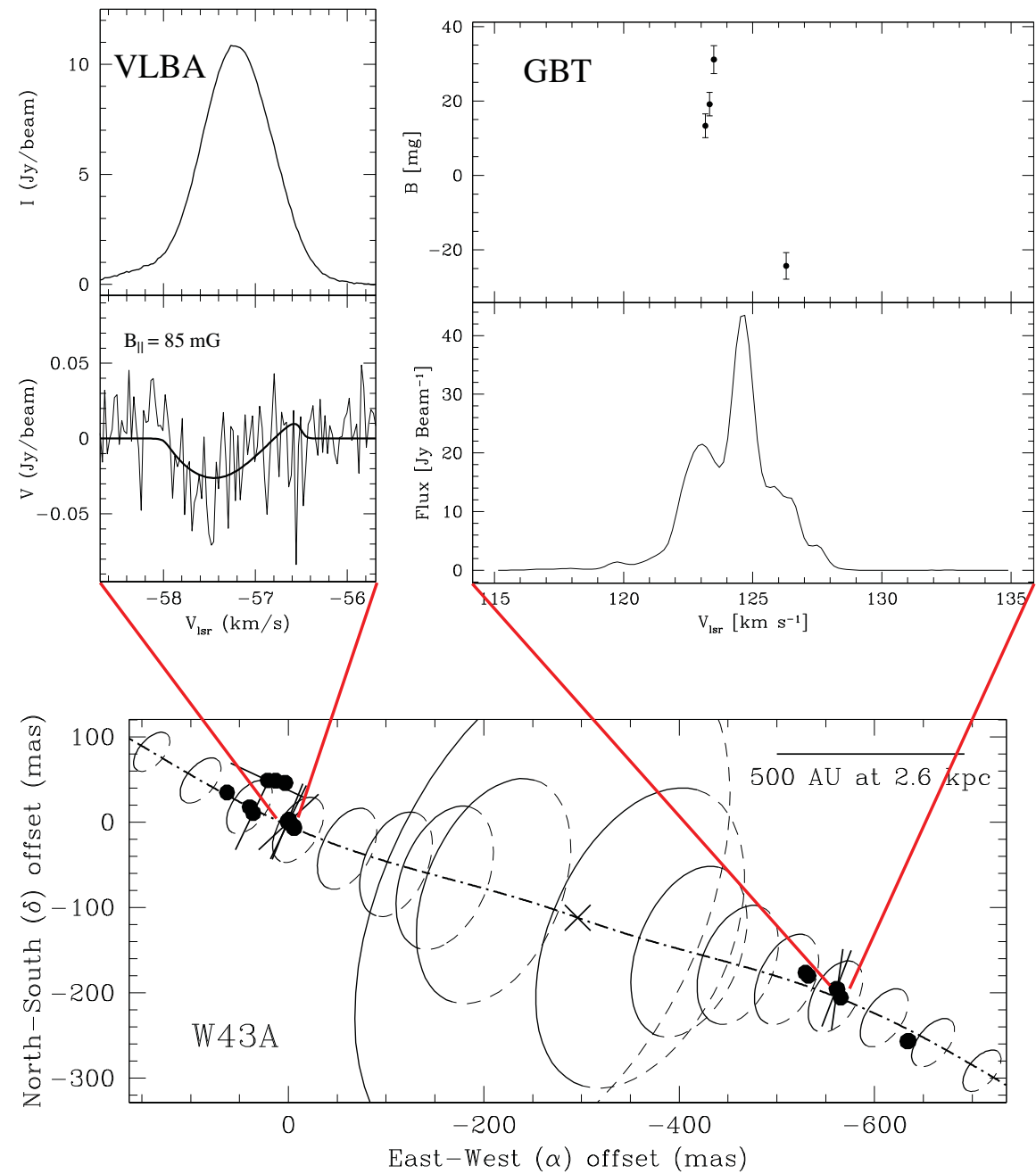

Figure 4. (top left) Total power (I) and V-spectrum for one of the $\mathrm{H}_{2} \mathrm{O}$ maser features in red-shifted lobe of the collimated jet of $\mathrm{W} 43 \mathrm{~A}$ including the best model fit of the $\mathrm{V}$-spectrum corresponding to a magnetic field (Vlemmings et al. 2006). (top right) Confirmation of the magnetic field from single-dish GBT observations in the blue-shifted side of the lobe. As expected for being toroidal, the magnetic field reverses sign across the blue-shifted masers (Amiri et al. 2010). (bottom) The $\mathrm{H}_{2} \mathrm{O}$ masers in the precessing jet (dashed-dotted line) of W43A (indicated by the cross) and the toroidal magnetic field of W43A. The vectors indicate the determined magnetic field direction, perpendicular to the polarization vectors, at the location of the $\mathrm{H}_{2} \mathrm{O}$ masers. The ellipses indicate the toroidal field along the jet, scaled with magnetic field strength $\propto r^{-1}$.

Finally, a recent direct measurement of the Zeeman effect on the surface of the Mira variable star $\chi C y g$ has been reported (Lèbre et al., this proceedings). The apparent field of a few Gauss would be reasonably consistent with the field measured throughout the envelope of other AGB stars.

\subsection{Post-AGB Stars and P-PNe}

Similar to the AGB stars, masers are the main source of magnetic field information of post-AGB and P-PNe, with the majority of observations focused on $\mathrm{OH}$ masers. These 


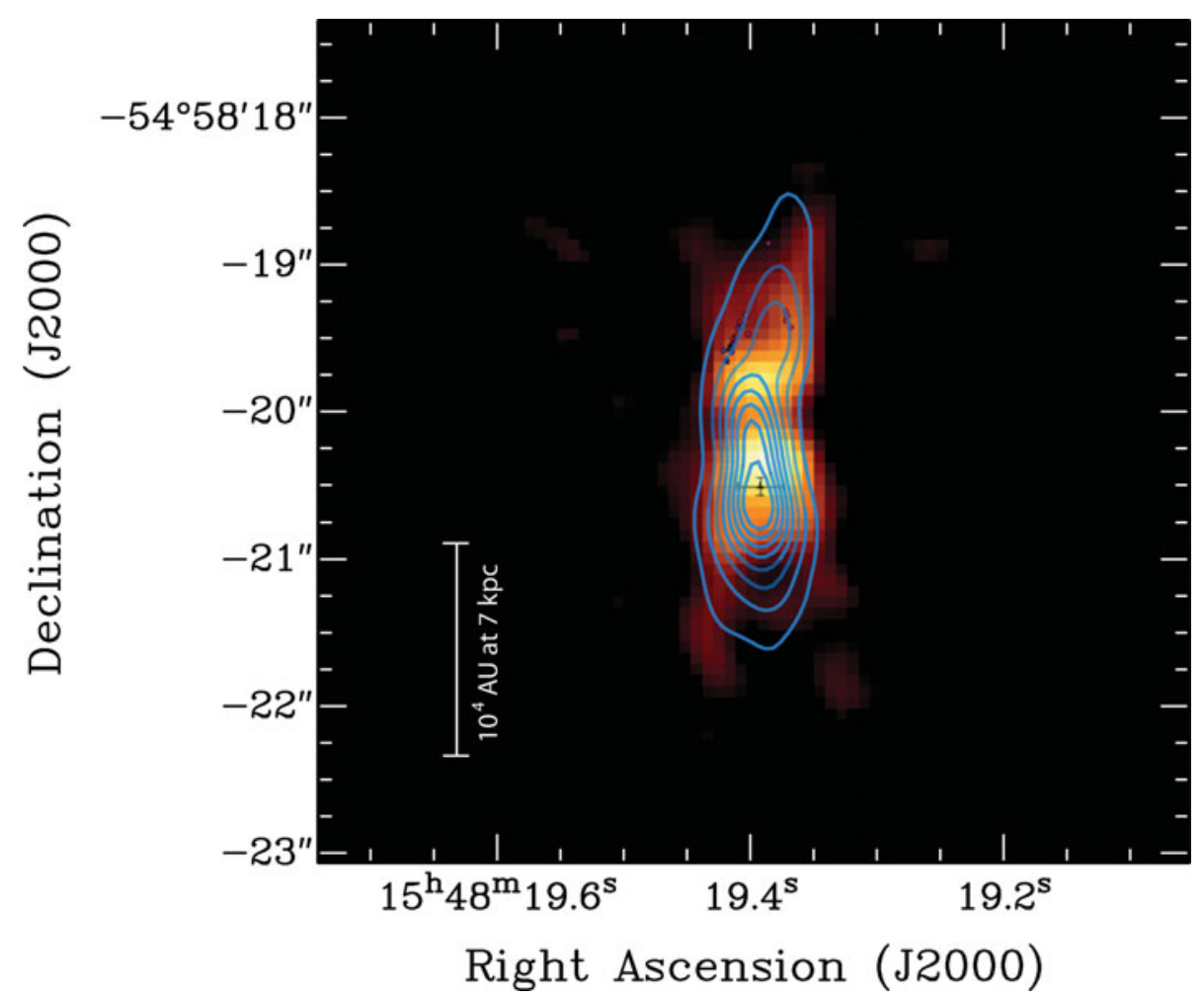

Figure 5. From Pérez-Sánchez et al. (2013). The synchrotron jet from IRAS 15445-5449 (contours) on a mid-infrared VLT image. Also indicated are the high-velocity maser features in the Northern lobe of the outflow.

have revealed magnetic field strengths similar to those of AGB stars (few $\mathrm{mG}$ ) and a clear large scale magnetic field structure (e.g. Bains et al. 2003).

The most promising results have come after the detection of the so-called 'waterfountain' sources. These sources exhibit fast and highly collimated $\mathrm{H}_{2} \mathrm{O}$ maser jets that often extend beyond even the regular $\mathrm{OH}$ maser shell. With the dynamical age of the jet of order 100 years, they potentially are the progenitors of the bipolar (P-)PNe. Although the masers are often too weak for a detection of the magnetic field, observations of the arch-type of the water-fountains, W43A, have revealed a strong toroidal magnetic field that is collimating the jet (Fig. 4 and Vlemmings et al. 2006).

Excitingly, the maser results have recently been strengthened by the direct detection of a synchrotron jet from the post-AGB star IRAS 15445-5449 (Pérez-Sánchez et al. 2013). This jet further proves the existence of strong collimating magnetic fields in the late stages of AGB evolution and strengthens the hypothesis that magnetic jets shape the bipolar PNe.

\subsection{Planetary Nebulae}

During the PN phase, masers are rare and weak and until now only the PN K3-35 has had a few $\mathrm{mG}$ magnetic field measured in its $\mathrm{OH}$ masers (Miranda et al. 2001). Fortunately, there are a few other methods of measuring PN magnetic fields. The field orientation in the dust of the nebula can be determined using dust continuum polarization observations and current observations seem to indicate toroidal fields, with the dust alignment likely occurring close to the dust formation zone (Sabin et al. 2007 and these proceedings). 
Table 1. Energy densities in AGB envelopes

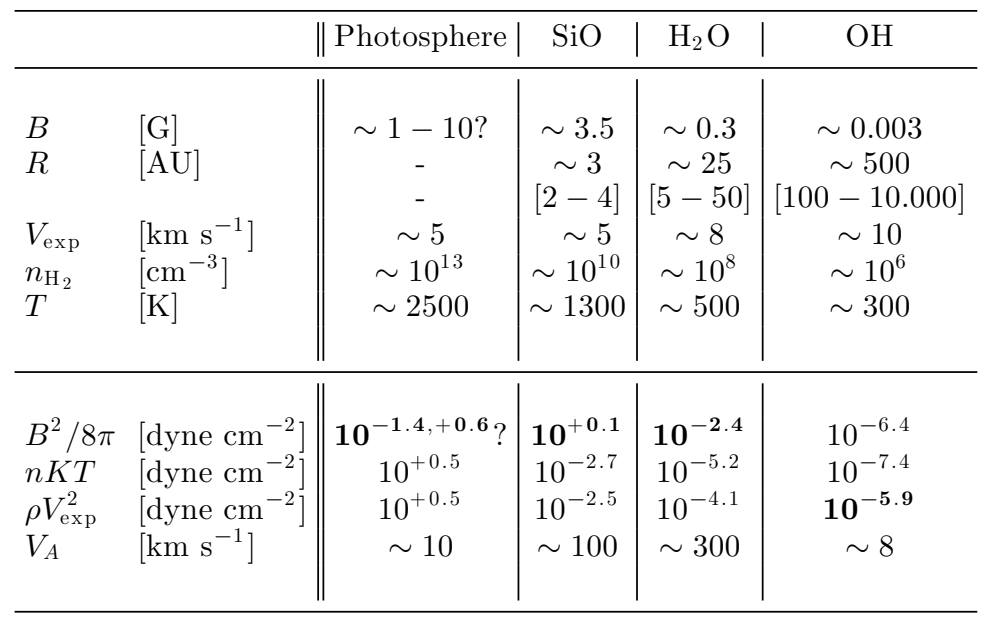

Faraday rotation studies are potentially also able to study the magnetic field in the interaction region between the interstellar medium and the stellar outflow.

In contrast to AGB stars, the central stars of PNe also show atomic lines that can be used to directly probe the magnetic fields on the surface of these stars. Despite earlier claims of the detection of a field of order several $\mathrm{kG}$, recent work shows that the previous detection was spurious and no direct detection has yet been made (Leone et al. 2011).

\section{Origin of the Magnetic Field}

Despite the strong observational evidence for evolved star magnetic fields, the origin of these fields is still unclear. In single stars, differential rotation between the AGB star core and the envelope could potentially result in sufficiently strong magnetic field (Blackman et al. 2001). However, as the energy loss due to a rotating magnetic field drag drains the rotation needed to maintain the field within several tens of years, an additional source of energy is needed (e.g. Nordhaus \& Blachman 2006). If AGB stars would be able to have a sun-like convective dynamo, magnetically dominated explosions could indeed result from single stars. Alternatively, the energy could be provided by the interaction with a circumstellar disk, although the origin of the disk is then another puzzle.

Another explanation for maintaining a magnetic field is the interaction between a binary companion or potentially a heavy planet, with common-envelope evolution providing paths to both magnetically as well as thermally driven outflows (Nordhaus \& Blackman 2006). A companion could be the cause of the precession seen in a number of water-fountain and (P-)PNe jets. However, to date, the majority of the stars with measured magnetic fields do not show any other indication of binarity. However, as shown by our recent ALMA observations of the detached shell carbon AGB star R Scl (Maercker et al. 2012), hidden binaries could be fairly commonplace.

\section{Effect of the Magnetic Field}

Until a more complete sample of magnetically active AGB stars, post-AGB stars and (P-)PNe is known, it is hard to observationally determine the effect of the magnetic field on these late stages of evolution. Starting with the AGB phase, a number of theoretical works have described the potential of magnetic fields in (at least partly) driving the 
stellar mass-loss through Alfvén waves (e.g. Falcetta-Gonçalves \& Jatenco-Pereira 2002), or through the creation of cool spots on the surface above with dust can form easier (Soker 1998). As current models of dust and radiation driven winds are still unable to explain especially the mass-loss of oxygen-rich stars, magnetic fields might provide the missing component of this problem, with tentative evidence already pointing to a relation between the magnetic field strength and mass-loss rate. The recently detected X-ray emission from two AGB stars could potentially be an indication of magnetic stellar activity (Ramstedt et al. 2012).

Other theoretical works have focused on the magnetic shaping of the stellar winds (e.g. Chevalier \& Luo 1994, García-Segura et al. 2005, Frank \& Blackman 2004). But to properly determine the possible effect of the magnetic fields, it is illustrative to study the approximate ratios of the magnetic, thermal and kinematic energies contained in the stellar wind. In Table.1 I list these energies along with the Alfvén velocities and typical temperature, velocity and temperature parameters in the envelope of AGB stars. While many values are quite uncertain, as the masers that are used to probe them can exist in a fairly large range of conditions, it seems that the magnetic energy dominates out to $\sim 50-100 \mathrm{AU}$ in the circumstellar envelope. This would correspond to the so-called 'launch' region of magneto-hydrodynamic (MHD) outflows, which typically extend to no more than $\sim 50 R_{i}$, with $R_{i}$ the inner-most radial scale of launch engine. A rough constraint on $R_{i}$ thus seems to be $\sim 1-2 \mathrm{AU}$, close to the surface of the star.

\section{Outlook}

While progress in studying the magnetic fields of evolved stars has been significant, a number of crucial questions remain to be answered. Several of these can be addressed with the new and upgraded telescopes in the near future. For example, the upgraded JVLA and eMERLIN will uniquely be able to determine the location of the masers in the envelope with respect to the central star, giving us, together with polarization observations, crucial information on the shape and structure of the magnetic field throughout the envelopes. ALMA will be able to add further probes of magnetic fields with for example high frequency masers (Pérez-Sánchez \& Vlemmings 2013) and CO polarization observations, significantly expanding our sample of stars with magnetic field measurements. With the ALMA sensitivity, polarization will be easily detectable even in short observations and thus, even if not the primary goal, polarization calibration should be done. Additionally, ALMA will, as already shown, be able to reveal the hidden binaries and through the study of the circumstellar chemistry investigate peculiarities that can be induced by a magnetically active star. The new low-frequency arrays, including the future Square Kilometer Array, can potentially be used to determine magnetic fields in the interface between the ISM and PNe envelopes through Faraday rotation observations and investigate emission from the interaction between the stellar outflow and magnetic field with a potential planetary companion.

With the advances in the search for binaries and the theories of common-envelope evolution and MHD outflow launching, the new observations will address for example:

- Under what conditions does the magnetic field dominate over e.g. binary interaction when shaping outflows?

- Are magnetic fields as widespread in evolved stars as they seem?

- What is the origin of the AGB magnetic field - can we find the binaries/heavy planets that might be needed?

- Is there a relation between AGB mass-loss and magnetic field strength? 


\section{References}

Amiri, N., Vlemmings, W., \& van Langevelde, H. J. 2011, A\&JA, 532, A149

Bains, I., Gledhill, T. M., Yates, J. A., \& Richards, A. M. S. 2003, MNRAS, 338, 287

Bujarrabal, V., Castro-Carrizo, A., Alcolea, J., \& Sánchez Contreras, C. 2001, A\&\&A, 377, 868

Chevalier, R. A. \& Luo, D. 1994, ApJ, 421, 225

Falceta-Gonçalves, D. \& Jatenco-Pereira, V. 2002, ApJ, 576, 976

Frank, A. \& Blackman, E. G. 2004, ApJ, 614, 737

García-Segura, G., López, J. A., \& Franco, J. 2005, ApJ, 618, 919

Herpin, F., Baudry, A., Thum, C., Morris, D., \& Wiesemeyer, H. 2006, A\& A, 450, 667

Herpin, F., Baudy, A., Josselin, E., Thum, C., \& Wiesemeyer, H. 2009, in IAU Symposium, vol. 259 of IAU Symposium, 47

Kemball, A. J., Diamond, P. J., Gonidakis, I., Mitra, M., Yim, K., Pan, K., \& Chiang, H. 2009, ApJ, 698, 1721

Kwok, S., Purton, C. R., \& Fitzgerald, P. M. 1978, ApJ, 219, L125

Leal-Ferreira, M. L., Vlemmings, W. H. T., Kemball, A., \& Amiri, N. 2013, A\&GA, 554, A134

Leone, F., Martínez González, M. J., Corradi, R. L. M., Privitera, G., \& Manso Sainz, R. 2011, ApJL, 731, L33

Maercker, M., Mohamed, S., Vlemmings, W. H. T., et al. 2012, Nature, 490, 232

Miranda, L. F., Gómez, Y., Anglada, G., \& Torrelles, J. M. 2001, Nature, 414, 284

Nordhaus, J. \& Blackman, E. G. 2006, MNRAS, 370, 2004

Nordhaus, J., Blackman, E. G., \& Frank, A. 2007, MNRAS, 376, 599

Pérez-Sánchez, A. F. \& Vlemmings, W. H. T. 2013, A\&A, 551, A15

Pérez-Sánchez, A. F., Vlemmings, W. H. T., Tafoya, D., \& Chapman, J. M. 2013, MNRAS in press, arXiv: 1308.5970

Ramstedt, S., Montez, R., Kastner, J., \& Vlemmings, W. H. T. 2012, AछA, 543, A147

Rudnitski, G. M., Pashchenko, M. I., \& Colom, P. 2010, Astron. Rep., 54, 400

Sabin, L., Zijlstra, A. A., \& Greaves, J. S. 2007, MNRAS, 376, 378

Soker, N. 1998, MNRAS, 299, 1242

Soker, N. 2002, MNRAS, 336, 826

Szymczak, M., Cohen, R. J., \& Richards, A. M. S. 2001, A\&\&A, 371, 1012

Vlemmings, W. H. T. 2007, in IAU Symposium, edited by Booth, R. S. Humphreys, E. M. L. \& Vlemmings, W. H. T., vol. 287 of IAU Symposium, 31

Vlemmings, W. H. T., Diamond, P. J., \& Imai, H. 2006, Nature, 440, 58

Vlemmings, W. H. T., Diamond, P. J., \& van Langevelde, H. J. 2002, A\&A A, 394, 589

Vlemmings, W. H. T., Humphreys. E. M. L. \& Franco-Hernández, R. 2011, ApJ, 728, 149

Vlemmings, W. H. T., van Langevelde, H. J., \& Diamond, P. J. 2005, A\&A, 434, 1029

Vlemmings, W. H. T., Ramstedt, S., Rao, R., \& Maercker, M. 2012, A\&A, 540, L3 